\section{-RÉSUMÉ}

\section{Tell tall}

M. Petrie and A. Williams went to work on an egg, or rather 519 of them, to test a prediction of life-history theory (Proc. R. Soc. Lond. B251, 127-131; 1993). The prediction is that if females gain in one way or another from mating with attractive males, they are likely to invest more in reproduction with such males. The eggs were those of peahens. They were laid by a total of 32 birds which had been split into groups of four, each of the groups being put into a pen with a single male. It turned out that significantly more eggs were produced for peacocks with larger masses of train feathers (mass was one of several possible measures of train elaboration). That females do indeed invest more in reproducing with more attractive males has been observed before, but not in a species such as peafowl in which males do not feed the female during courtship or help care for the offspring.

\section{Anybody there?}

\section{THE search for extraterrestrial} intelligence (SETI) may have more chance of success than those engaged on the project say (J. F. Kasting, D. P. Whitmire \& R. T. Reynolds Icarus 101, $108-128 ; 1993)$. For a planet to sustain life, it must be not so near the parent star that its water content is boiled and photolysed away; but nor should it be so far away that atmospheric carbon dioxide (necessary as a greenhouse gas) condenses to form clouds, shading the surface. With these twin conditions, the authors estimate the size of habitable zones around various types of star (fortunately, Venus and Mars fall outside the limits for the Sun). Hotter 'F' stars evolve too quickly for life to develop, but planets around cooler ' $M$ ' stars become tidally locked on their orbits, so that one hemisphere always looks out to cold outer space. $\mathrm{K}$ stars, however, seem to be even more favourable than the Sunlike G-stars, currently targeted by the SETI project, and should be included in the search, the authors argue.

\section{In the drink}

THE happiest experimental animals may be the strains of ethanol-sensitive mice that are indulged in their little weakness by G. S. Wand et al. (J. biol. Chem. 268 , 2595-2601; 1993). In cells in vitro, as well as in animals, ethanol intoxication represses the adenylylcyclase pathway with readily imaginable consequences on neurotransmission. GTP- and agoniststimulated adenylylcyclase activity in membranes of the mouse brain is found to fall. Wand and colleagues now show that a superabundance of the electric soup increases the expression of a pair of inhibitory G proteins, but the one responsible for stimulation is unaffected. A sobering thought!

\title{
Pick your poison
}

\section{R. J. Cava}

INVARIABLY, the first question colleagues ask if we haven't seen each other in a few months is "what's new in high- $T_{\mathrm{c}}$ ?". Like me, they remember the glory days when every week held the possibility that a new superconducting copper oxide would be discovered, and they would like to find out if there is anything new and exciting to hear. Although the level of worldwide effort in research into high-temperature superconductors is still reasonably high, the

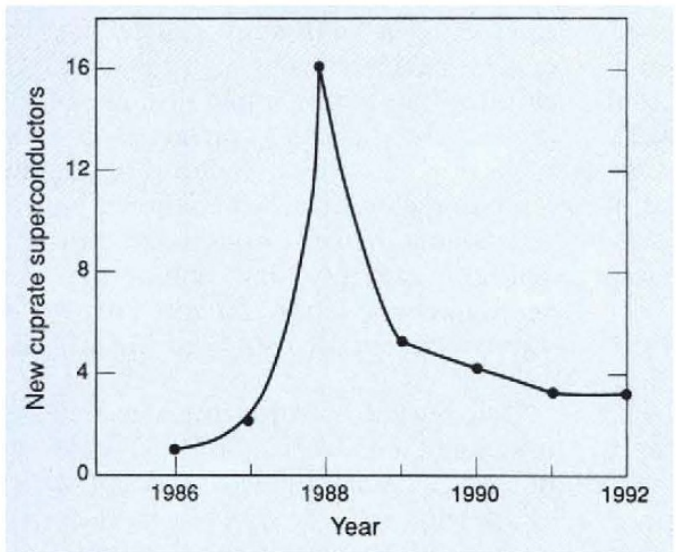

Rate of discovery of new, structurally or chemically distinct cuprate superconductors.

pace of dramatic discovery has slowed significantly in recent years. We find ourselves in a period of more conventional scientific process, as the rapidly maturing field advances by evolutionary rather than revolutionary steps. The announcement of the new 94-kelvin superconductor $\mathrm{HgBa}_{2} \mathrm{CuO}_{4+\delta}$ by $\mathrm{S}$. $\mathrm{N}$. Putilin and co-workers in this issue (Nature 362, 226-228; 1993) brings back some of the old flavour of expectation and potential that has been lacking in the superconducting-materials business of late.

Although commonly viewed as a revolution in solid-state physics, hightemperature superconductivity has been even more of a revolution in materials science. It has, after all, been driven from the very beginning by the discovery of new materials, not by either theoretical guidance or the understanding of the microscopic physics of the superconductivity. The new materials have followed a continuous path towards increasing chemical, synthetic and structural complexity in the past six years. It is no accident that the simplest materials were discovered earliest: the explosive increase in the superconducting onset temperature $\left(T_{\mathrm{c}}\right)$ that followed was a consequence of the explosive growth in knowledge in the materials-science community of the empirical chemical and structural factors necessary for hightemperature superconductivity to occur.

Unless your speciality is finding new superconducting materials, the number and complexity of known copper-oxidebased superconductors has reached the point where it is difficult to keep up. It is no help that many published reports claiming to reveal 'new' superconducting copper oxides are either completely wrong, or represent only the partial substitution of one atom for another in a trivial way in a superconductor that is already well known. Including the new $\mathrm{HgBa}_{2} \mathrm{CuO}_{4+\delta}$ superconductor announced in this issue, by my count there are 35 chemically or structurally unique copper-oxide superconductors known to date. The pace of discovery of those superconductors is shown in the figure. In the remarkable peak year of 1988,16 new cuprate superconductors were discovered, a majority of which were members of families of compounds based on bismuthoxide and thallium-oxide intermediary layers. The pace since 1989 has slowed to about four per year: almost without exception these have been more complex, more difficult to synthesize, and have lower transition temperatures than those found throughout 1988.

If viewed from a jaded perspective, the addition of one more superconductor to the list would not seem to make much difference. $\mathrm{HgBa}_{2} \mathrm{CuO}_{4+\delta}$ has, however, several qualities that distinguish it from other cuprate superconductors discovered of late. Most importantly, it is apparently easy to synthesize and structurally simple, bucking the now wellestablished trend towards difficulty and complexity. These qualities will greatly facilitate experimentation with it for potential applications. Secondly, its transition temperature of $94 \mathrm{~K}$ is very high, especially for a superconductor based, as it is, on a single copper layer. As the authors point out, members of this family based on double or triple copper layers, when synthesized and correctly processed, should have even higher transition temperatures, perhaps in the vicinity of $125 \mathrm{~K}$, which are accessible at present only in thallium-oxide-based materials. Current efforts in the development of bulk applications at liquidnitrogen temperature for superconductors centre around $\mathrm{YBa}_{2} \mathrm{Cu}_{3} \mathrm{O}_{7}$ and bismuth-oxide-based cuprates, with transition temperatures near $90 \mathrm{~K}$; in spite of their very high transition temperatures, 